\title{
An integrated flexible multifunctional sensing system for simultaneous monitoring of environment signals
}

\author{
Haoran Chen $^{1 \dagger}$, Zheng Lou ${ }^{1,2 \dagger}$ and Guozhen Shen ${ }^{1 *}$
}

\begin{abstract}
With the rapid development of portable devices and internet of things, the requirement of system wearability and integration accelerates the investigation of flexible multifunctional sensors. In this study, we developed an integrated flexible sensing system with four nanowire-based sensors and a Ni microwire-based temperature sensor. The four nanowirebased sensors are three kinds of photodetectors responding to lights with different wavelengths and a gas sensor. Due to the large surface volume ratio and considerable sub wavelength effect, all the nanowire-based sensors show good sensing response and excellent linear relationship between sensitivity and temperature. The as-fabricated flexible sensing system can simultaneously detect environmental parameters, including temperature change, light intensities from UV-Visible to near infrared regions, and harmful gas concentration. Our flexible multifunctional sensing system therefore opens up a new way for the emerging portable and wearable electronics.
\end{abstract}

Keywords: flexible device, nanowire, multifunctional sensing system, gas sensor, photodetector

\section{INTRODUCTION}

Tracking environmental conditions, including air particles, harmful gas concentration, humidity level, ambient temperature and ultraviolet (UV) radiation, offers valuable information for the prediction, treatment and management of chronic diseases [1-5]. Wearable devices are considered as one of the most feasible and effective ways for daily environmental monitoring [6,7]. Although the state-of-the-art developed wearable devices show superior performance, they usually have a single function to the target and can only work under specific conditions. Sometimes it cannot meet the requirements of miniaturization and the planar integration of the system. In this context, multifunctional sensing devices are more and more concerned by both industry and academia [8-10]. In addition to good multifunction, wearable sensors should also have high sensitivity, low power consumption, low cost, and good flexibility [11-14]. To achieve these characteristics, nanowires (NWs), which have excellent electrical properties, large surface area and compatibility with low-cost manufacturing process, are widely used to construct flexible devices [15-20]. For example, Fang et al. [21] recently reported a flexible photodetector based on InAs NWs, which showed great performance with high external quantum efficiency, responsivity, and detectivity. Kwon et al. [22] investigated a flexible gas sensor based on $\mathrm{ZnO} \mathrm{NWs}$ with excellent gas sensing performance and good mechanical flexibility. The device can still work normally without obvious degradation even after 10,000 dynamic bending tests with a bending radius of $5 \mathrm{~mm}$. Despite the impressive achievements of NWbased wearable sensors, multifunctional perception of various stimuli is still the ideal choice to expand its practical application.

Herein, we proposed a multifunctional system integrated with five kinds of flexible sensors based on different NWs and Ni microwires. This coplanar system has good flexibility, can respond to a variety of stimuli (such as gas, light and temperature) under outdoor conditions, and has high linearity, high resolution and no time delay. Our work represents a good example to integrate different types of flexible sensors to achieve highly specific and sensitive environmental signal detection, which could meet the wearable electronic requirements for real-time understanding of environmental conditions under different stimuli. It exploits a new strategy for the development of the next generation robust systems based on

\footnotetext{
${ }^{1}$ State Key Laboratory for Superlattices and Microstructures, Institute of Semiconductors, Chinese Academy of Sciences \& Center of Materials Science and Optoelectronic Engineering, University of Chinese Academy of Sciences, Beijing 100083, China

${ }^{2}$ State Key Laboratory of Transducer Technology, Aerospace Information Research Institute, Chinese Academy of Sciences, Beijing 100080, China

${ }^{\dagger}$ These two authors contributed equally to this work.

* Corresponding author (email: gzshen@semi.ac.cn)
} 
multifunctional wearable sensors.

\section{EXPERIMENTAL SECTION}

\section{Synthesis of CdS NWs}

CdS NWs were synthesized via a facile hydrothermal method. Firstly, $2 \mathrm{mmol}$ of $\mathrm{Cd}\left(\mathrm{NO}_{3}\right)_{2} \cdot 4 \mathrm{H}_{2} \mathrm{O}$ was dissolved in ethylenediamine (EDA, $30 \mathrm{~mL}$ ), and $4 \mathrm{mmol}$ of $\mathrm{CH}_{4} \mathrm{~N}_{2} \mathrm{~S}$ was added and stirred at room temperature for $30 \mathrm{~min}$. Then, the mixed solution was transferred to a 50-mL Teflon-lined stainless steel autoclave, and heated at $200^{\circ} \mathrm{C}$ for $24 \mathrm{~h}$. After the autoclave was naturally cooled down to room temperature, the product was taken out and washed with ultra-pure water and ethanol for several times.

\section{Synthesis of ZnO NWs}

$\mathrm{ZnO}$ NWs were prepared via the electrospinning process. Typically, $0.1 \mathrm{~g}$ of zinc acetate $\left(\mathrm{Zn}\left(\mathrm{CH}_{3} \mathrm{COO}\right)_{2} \cdot \mathrm{H}_{2} \mathrm{O}\right)$ was dissolved in $50 \mathrm{~mL}$ of $\mathrm{N}, \mathrm{N}$-dimethylformamide and $50 \mathrm{~mL}$ of ethanol. Subsequently, $1 \mathrm{~g}$ of polyvinylpyrrolidone (PVP, molecular weight $=1,300,000$ ) was added under stirring for $1 \mathrm{~h}$ to achieve a transparent solution. In order to achieve an electrospinning process, the resulting solution was loaded into a syringe using an 8-gauge needle. The feeding rate was $0.2 \mathrm{~mL} \mathrm{~h}^{-1}$, and an electrical field was applied as high as $1 \mathrm{kV} \mathrm{cm}{ }^{-1}$ between the tip of the syringe and the collector. Then, the nanofibers were collected on the surface of the collector and calcined in a muffle furnace at a temperature of $650^{\circ} \mathrm{C}$ for $4 \mathrm{~h}$, and then were ground into powder.

\section{Synthesis of SnS NWs}

To synthesize SnS NWs via the physical vapor deposition (PVD) method, an alumina boat which contained $0.15 \mathrm{~g}$ of high-purity SnS powder (99.5\%, Alfa) was placed at the center of a small quartz tube in a tube furnace. A Si wafer coated with 10-nm-thick Au catalysts was placed $16 \mathrm{~cm}$ downstream away from the SnS powder to collect SnS NWs. After the tube furnace was flushed with Ar gas for $30 \mathrm{~min}$, the tube furnace temperature was raised to $700^{\circ} \mathrm{C}$ in $35 \mathrm{~min}$ and then kept at $700^{\circ} \mathrm{C}$ for $30 \mathrm{~min}$. During the growth process, the Ar flow rate was maintained at a rate of $100 \mathrm{sccm}$. When the deposition process was ended, the tube furnace was naturally cooled down to room temperature under Ar flow.

\section{Synthesis of $\mathrm{SrGe}_{4} \mathrm{O}_{9} \mathrm{NWs}$}

$\mathrm{SrGe}_{4} \mathrm{O}_{9}$ NWs were prepared by the hydrothermal method. First, $2 \mathrm{mmol}$ of $\mathrm{Sr}\left(\mathrm{CH}_{3} \mathrm{COO}\right)_{2} \cdot 1 / 2 \mathrm{H}_{2} \mathrm{O}$ and
$8 \mathrm{mmol}$ of $\mathrm{GeO}_{2}$ were added to deionized water $(30 \mathrm{~mL})$. After stirring for $60 \mathrm{~min}$, the mixture was transferred to a Teflon-lined autoclave, and then heated to $180^{\circ} \mathrm{C}$ for $24 \mathrm{~h}$, followed by natural cooling to room temperature. After being washed thrice and dried at $80^{\circ} \mathrm{C}$ for $12 \mathrm{~h}$, the $\mathrm{SrGe}_{4} \mathrm{O}_{9}$ NWs were obtained.

\section{Fabrication of flexible multifunctional sensing system}

Multi-mask photolithography was utilized to fabricate the integrated system. First, Ni electrode layer $(100 \mathrm{~nm})$ was patterned on a polyimide (PI) substrate after a photolithography, thermal evaporation and lift-off process to build the temperature sensor. With a similar process, the source and drain electrodes $(\mathrm{Cr} / \mathrm{Au} 10 / 50 \mathrm{~nm})$ of photodetectors and gas sensors were then deposited on the flexible substrates. After that, four kinds of NWs were spin-coated on the corresponding electrodes. A wearable multifunctional integrated sensing system was thus successfully fabricated on the flexible PI substrate.

\section{Characterizations}

Scanning electron microscopy (SEM, Zeiss Supra55 (VP)) and powder X-ray diffraction (XRD, Rigaku D/Max-2550, $\lambda=1.5418 \AA$ ) analysis were employed to measure the morphologies and crystallinities of the four NWs, respectively. A probe station supported with a semiconductor characterization system (Keithley 4200-SCS) was utilized to measure the electronic characteristics of the four sensors. The light illumination source was a power-adjustable light source capable of outputting homogeneous light.

\section{RESULTS AND DICUSSION}

Fig. 1a shows the schematic illustration of the designed flexible multifunctional sensing system for environmental monitoring. The entire system consists of a temperature sensor, three kinds of photodetectors and a gas sensor. All these components are integrated on a PI film via a conventional photolithography process. Fig. $1 b, c$ show the photographs of the fabricated device, which exhibits good flexibility and could be attached to human body.

SEM images of the NWs used as sensing elements for different types of sensors are depicted in Fig. 1d. For all the four kinds of semiconducting NWs, they possess obvious one-dimensional (1D) nanostructures with smooth and clean structures. Typical diameters of the NWs range from $\sim 50$ to $200 \mathrm{~nm}$, depending on the techniques used to synthesize the NWs. XRD was used to check the purity and phase structure of the NWs and the corresponding results are depicted in Fig. 1e. For all the 
a

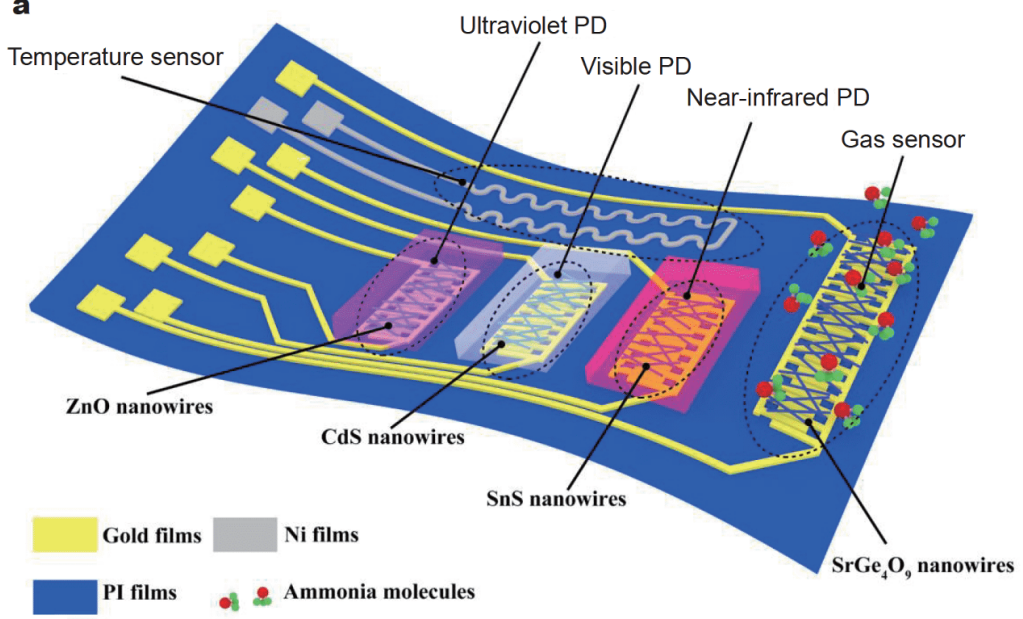

b

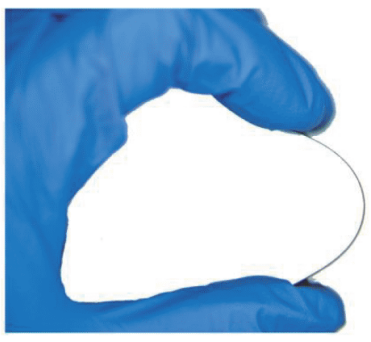

c

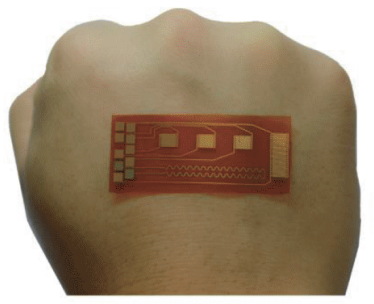

d
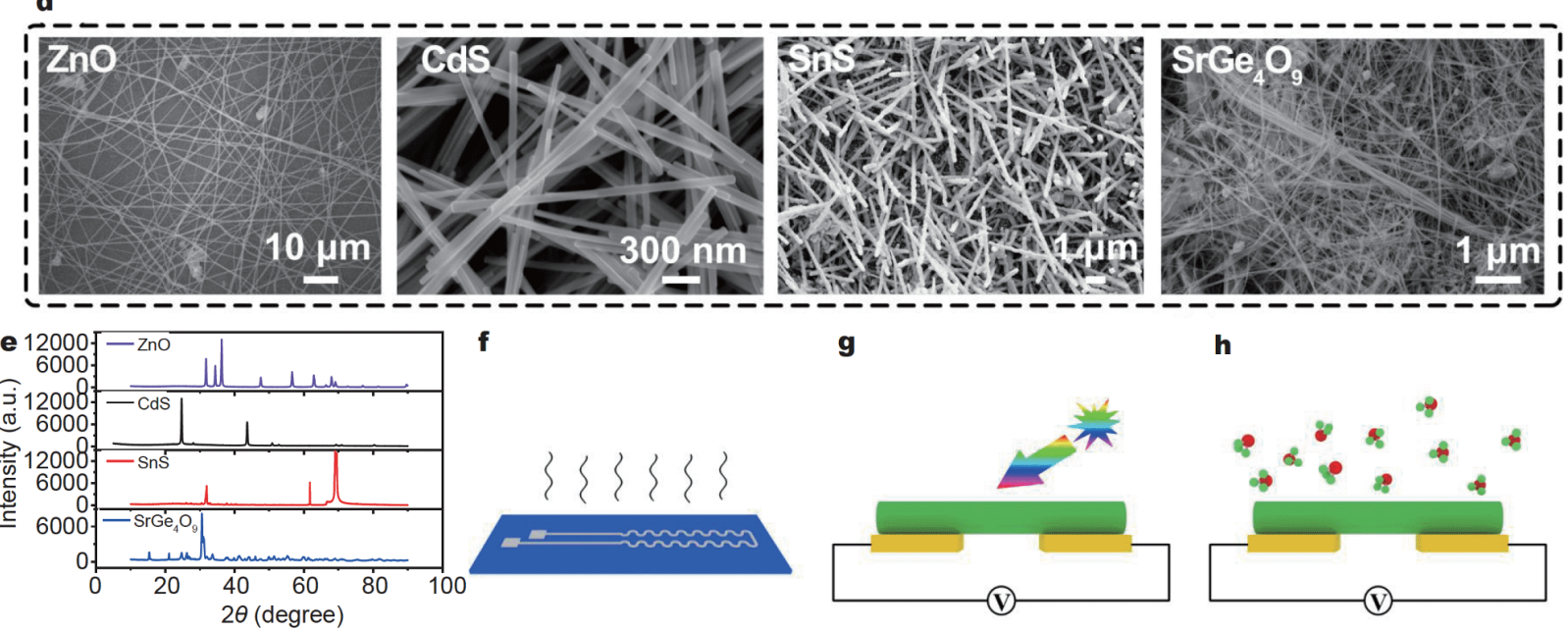

g

h
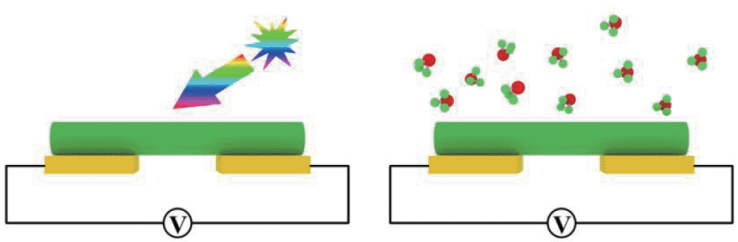

Figure 1 (a) A schematic of five pixels of a multimode wearable sensor capable of mapping five separate stimuli. PD: photodetector. (b) A photograph of the multifunctional device on a PI substrate with good flexibility. (c) A photograph of the matrix attached to the back of the hand. (d) SEM images and (e) XRD patterns of the four different sensing materials, including $\mathrm{ZnO}, \mathrm{CdS}, \mathrm{SnS}, \mathrm{SrGe}_{4} \mathrm{O}_{9}$. Schematic device structure of the three different kinds of sensors, (f) temperature sensor, (g) photodetector and (h) gas sensor.

samples, XRD data reveal that they are pure phases and can be indexed into wurtzite $\mathrm{ZnO}$ (JCPDS Card No. 361451), wurtzite CdS (JCPDS Card No. 41-1049), orthorhombic SnS (JCPDS Card No. 39-0354) and hexagonal $\mathrm{SrGe}_{4} \mathrm{O}_{9}$ (JCPDS Card No.14-0029), respectively. Fig. If$\mathrm{h}$ demonstrate the device structures of the temperature sensor, the photodetectors, and the gas sensor. The resistance-based temperature sensor was realized by fabricating waved $\mathrm{Ni}$ metal microwires as the sensing materials for detecting environmental temperature signals (Fig. 1f). The photodetectors based on three different kinds of NWs (ZnO NWs, CdS NWs and SnS NWs) could achieve different light spectrum detection from ultraviolet, visible to near-infrared light (Fig. 1g, respec- tively). $\mathrm{SrGe}_{4} \mathrm{O}_{9} \mathrm{NW}$-based gas sensor could adsorb ammonia molecules to realize real-time monitoring of toxic and harmful gases in the environment (Fig. 1h).

Because of the small size and large specific surface area, photodetectors based on 1D NWs have better performance compared with their thin film or bulk counterparts $[23,24]$. Surface carrier trapping has an important effect on the transport and photoconductivity of NWs. The schematic of the photodetection mechanism of NWs is shown in Fig. 2a. The process of oxygen adsorption and desorption in the dark and under light illumination is generally considered to be related to the free carriers produced. When exposed to air, the surface of NWs adsorbs oxygen molecules, which capture free electrons. A 

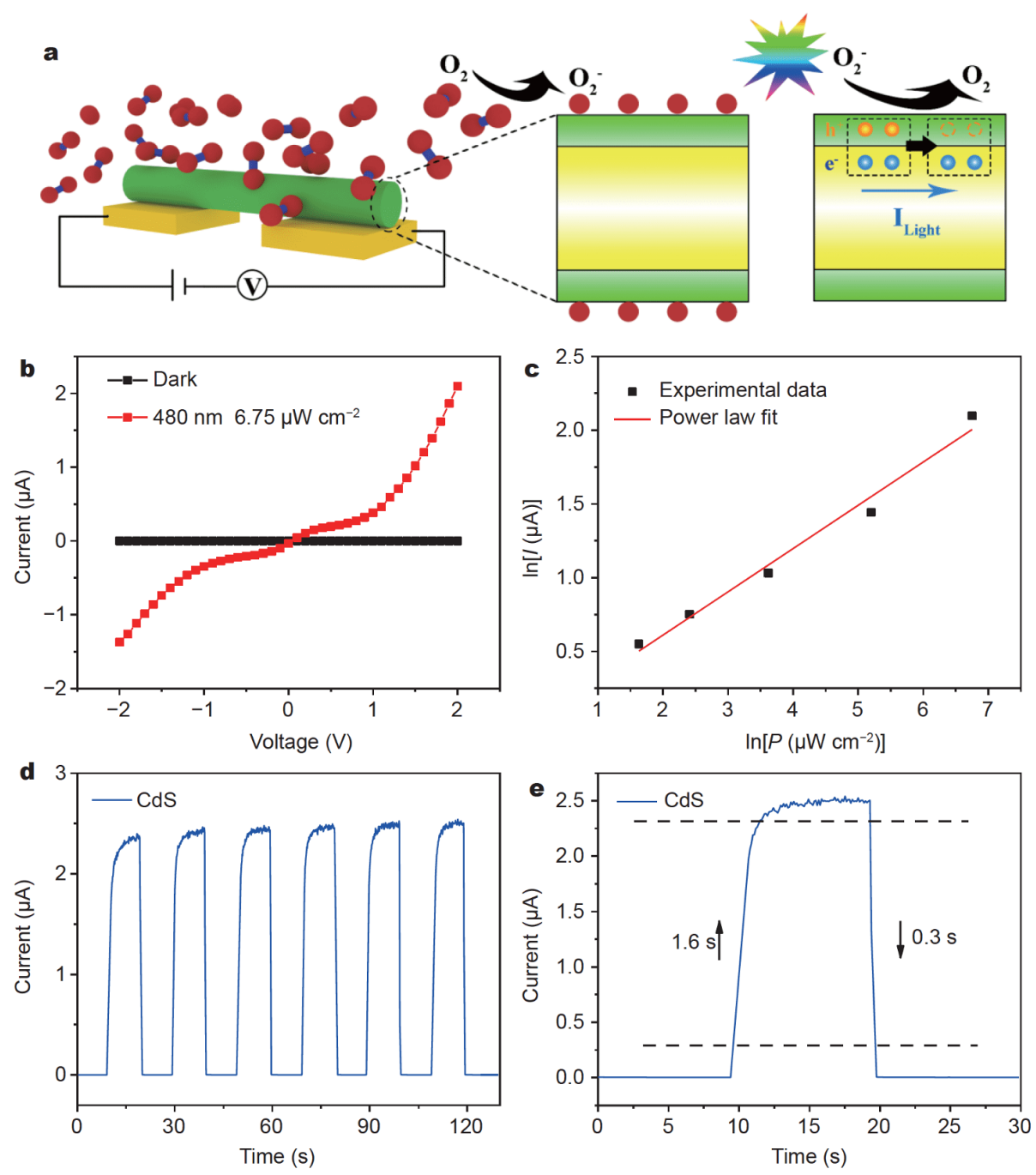

Figure 2 (a) Sensing mechanism of the NW-based photodetector. (b) $I$ - $V$ curves of the CdS NW-based photodetector in dark and under $480 \mathrm{~nm}$ light illumination, respectively. (c) The dependence of photocurrent on light intensity $\left(I \sim P^{1.01}\right)$. (d) Transient photoresponse properties of the photodetector with the light intensity of $6.75 \mu \mathrm{W} \mathrm{cm}{ }^{-2}$. (e) Enlarged part of the middle cycle of (d).

depletion layer is then formed on the surface of the NWs, resulting in low conductivity. As the device is irradiated by the incident light, the electron-hole pair is generated, and the unpaired electrons leave because the holes are easily trapped on the surface [25]. Under the action of an external electric field, the conductivity of the NWs increases, resulting in the decrease of depletion barrier thickness and the increase of free carrier concentration.

The photoresponse characteristics of CdS NW-based photodetector were studied in detail, and the corresponding results are shown in Fig. $2 b-e$. Fig. $2 b$ shows the $I-V$ curves of the device in the dark and under $480 \mathrm{~nm}$ light illumination, respectively. Obvious response to the visible light was observed for the device. Under the bias voltage of $2 \mathrm{~V}$, the current increases from $\sim 10 \mathrm{fA}$ to $2 \mu \mathrm{A}$ (light intensity of $6.75 \mu \mathrm{W} \mathrm{cm} \mathrm{cm}^{-2}$ ). A power law of the photocurrent versus light intensity is described in Fig. $2 \mathrm{c}$ as $I=A P^{\theta}$, where $I, A, P, \theta$ correspond to the photocurrent, a constant, the light intensity and the empirical value, respectively. From Fig. 2c, it can be seen that the light intensity and the photocurrent are highly dependent, resulting in the value of $\theta$ at $2 \mathrm{~V}$ bias voltage of 1.01 $\left(I \sim P^{1.01}\right)$, which proves that CdS NWs possess excellent photocurrent capability to visible light.

Reproducibility and response speed are critical factors to evaluate the performance of the photodetector, especially when used in optical communication, imaging and so on. By periodically turning the light on and off, we 
measured the transient photoresponse of the CdS-based photodetector and the corresponding result was shown in Fig. 2 d. For the given six light on/off periods, the photocurrent does not show obvious change, indicating that the device has good repeatability and stability under light irradiation. The photoresponse speed and recovery speed of the device can be deduced from a single light on/off curve, as demonstrated in Fig. 2e. The rise and fall times were thus measured to be about 1.6 and $0.3 \mathrm{~s}$, respectively, comparable to the previous reported data [16].

In addition to the visible light, our system can also monitor UV light in real-time through the integrated $\mathrm{ZnO} \mathrm{NW}$-based photodetector. The photoresponse measurements of the $\mathrm{ZnO}$-based devices under $350 \mathrm{~nm}$ UV light were systematically investigated, as demonstrated in Fig. 3. Fig. 3a shows the typical $I$ - $V$ curves of the $\mathrm{ZnO}$-based devices under UV illumination with the power intensity of $4.46 \mu \mathrm{W} \mathrm{cm} \mathrm{cm}^{-2}$ and in the dark, respectively. The good linearity of the $I-V$ curve indicates that the $\mathrm{ZnO} \mathrm{NWs}$ channel materials contact well with the metal electrode. When the light was exposed on the device, a significant increase in current was observed.
With the increase in light intensity, the photocurrent also increased accordingly (Fig. 3b). As shown in Fig. 3b, the dependence of photocurrent on the incident light intensity $(P)$ at the $V_{\text {bias }}=2 \mathrm{~V}$ can be accurately fitted by the power-law relationship of $I \sim P^{0.71}$. The result shows the nonlinear power dependence of the net photocurrent on the power density, and reveals that there are complex processes of electron hole generation, trapping and recombination in the NW channel. In order to study the ability of the $\mathrm{ZnO} \mathrm{NW}$ photodetector to track the changed light signal, the time-resolved photocurrent measurement was carried out by periodically modulating the $360 \mathrm{~nm}$ UV light on and off. Obviously, Fig. 3c depicts the photocurrent trace of the device at a bias voltage of $2 \mathrm{~V}$ with a light intensity of $4.46 \mu \mathrm{W} \mathrm{cm}$. The effective switching behavior indicates that the $\mathrm{ZnO} \mathrm{NW}$ device has good periodicity and stability with a rise time of $35.69 \mathrm{~s}$ and a fall time of $29.93 \mathrm{~s}$ (Fig. 3d). The relatively slow response of the $\mathrm{ZnO} \mathrm{NW}$ device was often observed for metal oxide NWs and had been extensively reported [26].

Infrared photodetector represents another important optoelectronic device and has attracted considerable re-
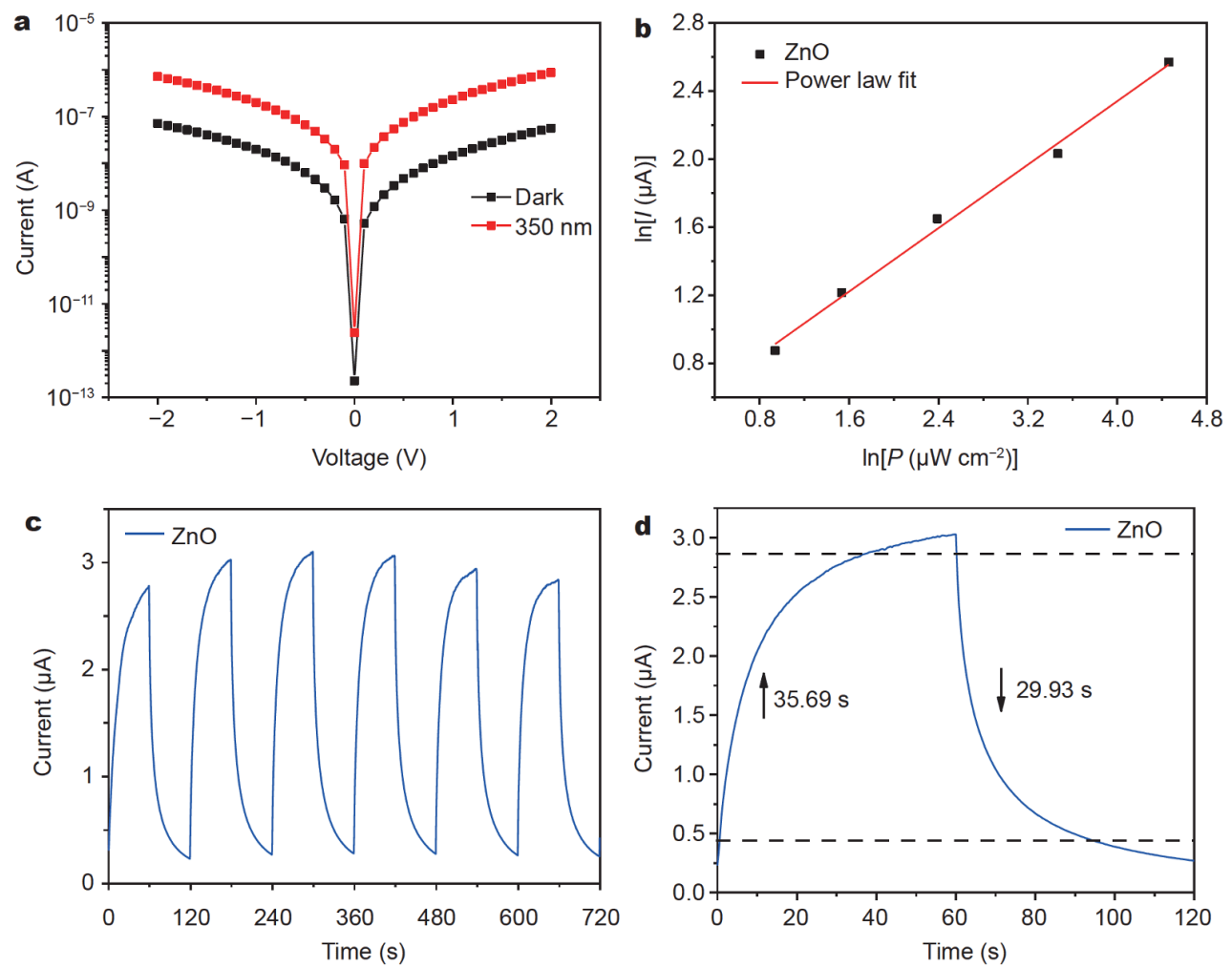

Figure 3 (a) $I-V$ characteristics of the $\mathrm{ZnO}$ NW-based photodetector under UV light illumination. (b) The relationship between photocurrent and light intensity $\left(I \sim P^{0.71}\right)$. (c) The transient photoresponse curves of the ZnO NW-based photodetector under UV light illumination. (d) The single response and recovery curve of the $\mathrm{ZnO}$-based photodetector. 
search attention in recent years. To endow our integrated system with the ability to detect infrared light, we integrated SnS NW-based devices into the flexible system, as $\mathrm{SnS}$ was an ideal candidate for mid near infrared (NIR) photodetectors because of its mid NIR direct band gap of $1.3 \mathrm{eV}$ and high absorption coefficient larger than $10^{4} \mathrm{~cm}^{-1}$. Fig. 4a shows the optical image of the measurement of the SnS-based device in the fabricated flexible integrating system. As shown in Fig. 4b, under constant source drain bias, the output current of the device obviously increases with the irradiation of incident infrared light $(808 \mathrm{~nm})$. The time-resolved photoresponse measurements were performed by periodically modulating the light illumination (Fig. 4c, d). The effective switching characteristics show that the SnS NW detector has good periodicity and stability. The rise and fall times can be determined from a single light on/off curve, as shown in Fig. $4 \mathrm{~d}$. The rise time and the decay time are 0.93 and $2.91 \mathrm{~s}$, respectively, indicating the fast response of the SnS-based photodetector.

As a basic physical parameter, temperature changes in time and space can be used to monitor physiological activities, especially the heat transfer between the environment and the biological tissues [27]. Wearable temperature detection requires the sensor to have enough sensitivity and accuracy, which can directly attach to the non planar skin surface with different posture and motion, and continuously measure the minimum user perception. To measure the temperature in real-time and to eliminate the temperature effect on other sensors, we integrated a $\mathrm{Ni}$ microwires-based temperature sensor on the fabricated integrated sensing system (Fig. 5a). The temperature sensor was fabricated via the photolithograph process, followed with the deposition of $\mathrm{Ni}$ on the patterned structure to form the waved $\mathrm{Ni}$ microwires. Fig. $5 \mathrm{~b}$ shows the change of current with changed temperature from 25 to $100^{\circ} \mathrm{C}$. With increased temperature, an increase in current was observed at a bias voltage of $1 \mathrm{~V}$. It indicated an obvious negative temperature coefficient (NTC) feature [28]. Due to the well-defined linear normalized resistance $v$ s. temperature relationship, the sensitivity of the temperature sensor was estimated to be about $3.4 \Omega /{ }^{\circ} \mathrm{C}$.

In order to eliminate the effect of temperature on other
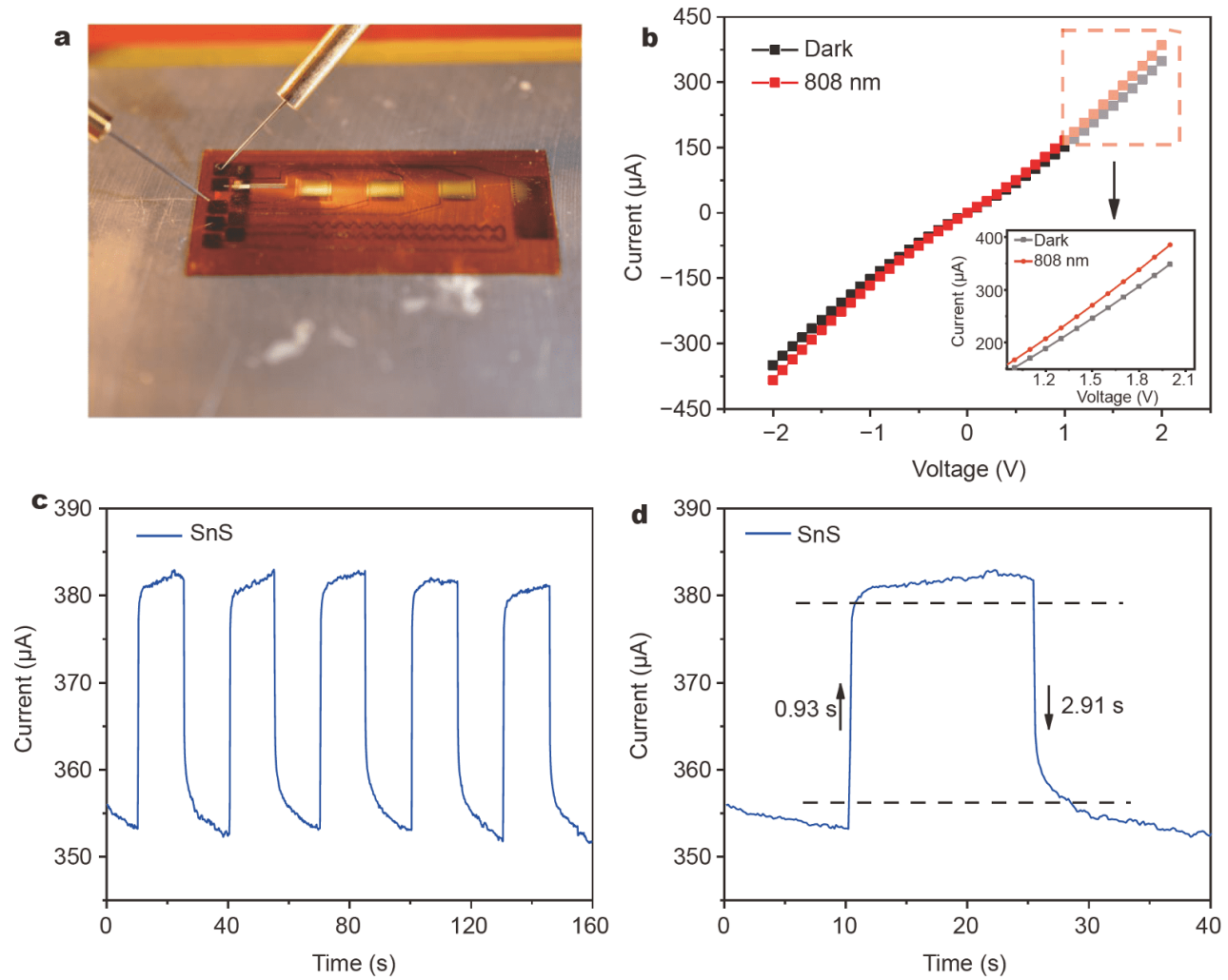

Figure 4 (a) Optical image of the measurement of the SnS-based device in the fabricated flexible integrating system. (b) $I$ - $V$ curves of the photodetector measured under NIR light illumination and in the dark, respectively. Inset is the enlarged part from 0.9-2.1 V. (c) The photoreponse curve of the SnS-based photodetector under $808 \mathrm{~nm}$ light with $2 \mathrm{~V}$ bias voltage. (d) Response and recover time of the device. 

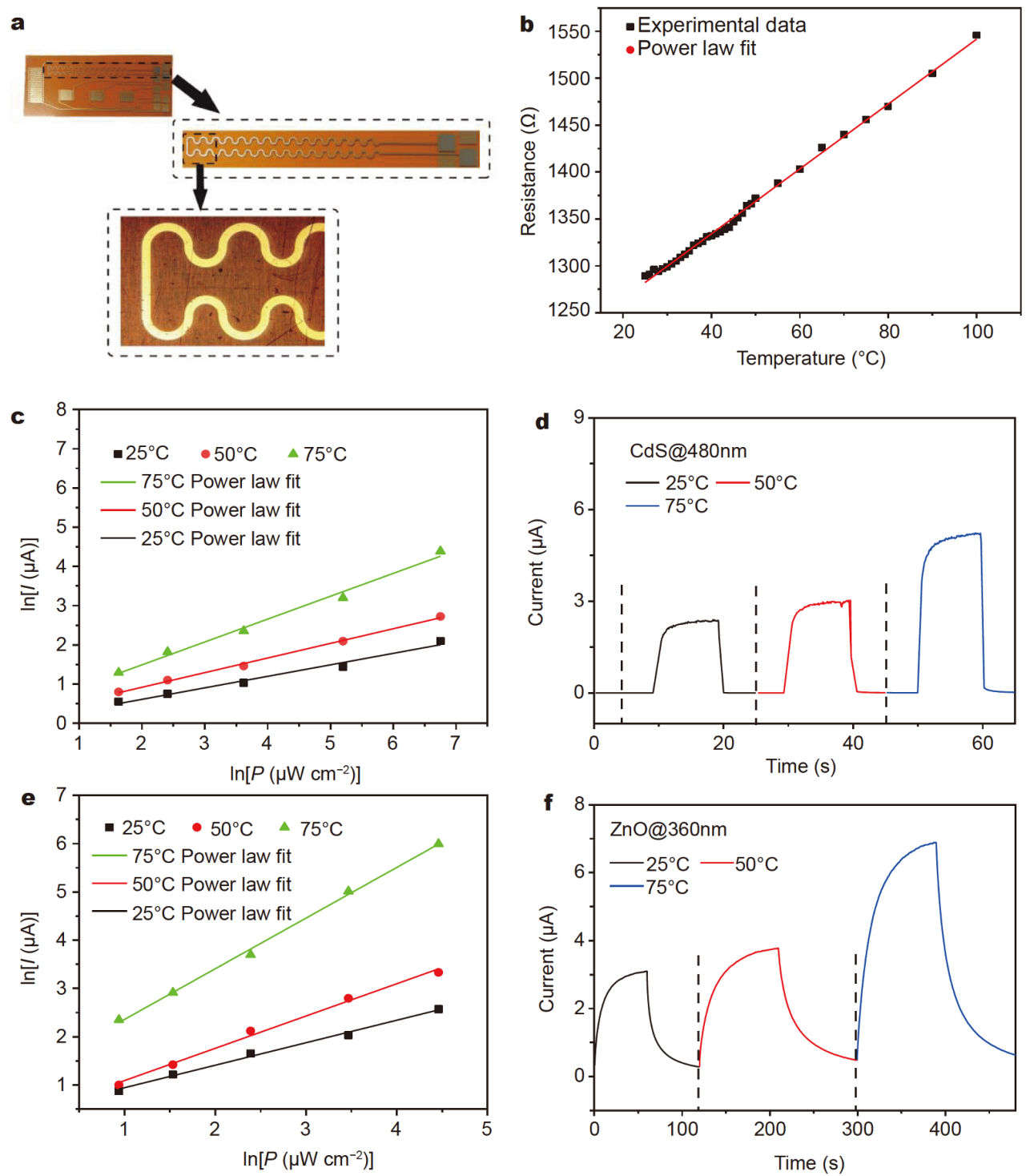

Figure 5 (a) Photographs and optical image of the flexible temperature sensor. (b) The relationship between resistance and temperature of the Ni microwires-based sensor. (c) Power dependence of the CdS NW photodetector, (d) dynamic photoresponse curves of the CdS NW photodetector, (e) power dependence of the $\mathrm{ZnO} \mathrm{NW}$ photodetector, (f) dynamic photoresponse of the $\mathrm{ZnO} \mathrm{NW}$ photodetector under different environment temperatures $\left(25\right.$ to $\left.75^{\circ} \mathrm{C}\right)$.

type sensors, the relationship between temperature and photodetectors was then studied by measuring the photoresponse of the photodetectors at different temperatures, as shown in Fig. 5c-f. The dependence of photocurrent on the incident light intensity under different temperatures at the bias voltage of $2 \mathrm{~V}$ is depicted in Fig. 5c. A linear relationship between the photocurrent and the changed light intensity was observed, which was also confirmed by the result in Fig. 5d. Moreover, the response and recovery time of the device did not show obvious change with the changed temperature. Similar results were also obtained on the $\mathrm{ZnO} \mathrm{NW}$-based photodetector, as shown in Fig. 5e, f. Briefly, temperature has a linear effect on the photocurrent of the $\mathrm{ZnO}$ devices. With the increase of temperature, the photocurrent of the device increases at different light intensities. Based on these results, it is possible to compensate the effect of temperature on other sensors by circuit design for practical applications.

Highly toxic gases including ammonia $\left(\mathrm{NH}_{3}\right)$ not only have a negative impact on the surrounding environment, but also are harmful to public health. By integrating 
flexible gas sensors, our wearable multifunctional system could be used to successfully detect the $\mathrm{NH}_{3}$ gas at room temperature [29]. The proposed sensing mechanism of the $\mathrm{SrGe}_{4} \mathrm{O}_{9} \mathrm{NW}$-based gas sensors in air and $\mathrm{NH}_{3}$ is shown in Fig. 6a. When exposed to air, oxygen molecules were physically adsorbed on the surface of the $\mathrm{SrGe}_{4} \mathrm{O}_{9}$ NWs through gaining electrons in the trapping conduction band, forming $\mathrm{O}^{-}$. As the depletion layer widened, the measured resistance of the sensor increased. When the sensor was exposed to $\mathrm{NH}_{3}$, the $\mathrm{O}^{-}$reacted with $\mathrm{NH}_{3}$ as follows[30]:

$2 \mathrm{NH}_{3}+5 \mathrm{O}^{-}=2 \mathrm{NO}+3 \mathrm{H}_{2} \mathrm{O}+5 \mathrm{e}^{-}$.

The narrow depletion layer resulted in the decrease of the measured resistance of the sensor.

The repeatability of the $\mathrm{SrGe}_{4} \mathrm{O}_{9} \mathrm{NW}$ sensor toward $100 \mathrm{ppm} \mathrm{NH}_{3}$ gas is shown in Fig. 6b. Four exposure/ recovery cycles were performed under the same conditions. No significant changes in response amplitude and recovery time were observed, which indicated that our $\mathrm{SrGe}_{4} \mathrm{O}_{9} \mathrm{NW}$ gas sensor had excellent stability. Fig. $6 \mathrm{c}$ shows the sensing performance of the $\mathrm{SrGe}_{4} \mathrm{O}_{9} \mathrm{NW}$ to $\mathrm{NH}_{3}$ gas at room temperature with various concentrations of 20-500 ppm, respectively. With increased $\mathrm{NH}_{3}$ concentrations, the responsivity was also gradually improved. For instance, when exposed to $100 \mathrm{ppm} \mathrm{NH}_{3}$ gases, the responsivity was 2.48 , while it was about 8 with the $\mathrm{NH}_{3}$ concentration of $500 \mathrm{ppm}$. For oxide gas sensor, temperature will affect the sensitivity of the device. As shown in Fig. 6d, increasing the temperature from 25 to $75^{\circ} \mathrm{C}$, the responsivity of the device decreases. As a result, for the gas sensor, the effect of temperature on its performance can also be compensated by circuit design to accurately measure the $\mathrm{NH}_{3}$ gas. The humidity effects on the sensing performance of the five devices were also studied. Fig. S1 shows the sensitive retention performance of the devices under different relative humidity (RH). The definition of sensitive retention is the ratio of sensitivity in atmosphere to the sensitivity with different $\mathrm{RH}$. These results show that the sensitivity is maintained from $80 \%-$ $95 \%$ of its original value of the five devices even under 95\% RH (the ambient RH is about 40\%). Therefore, for our sensing system, we can conclude that the effect of humidity on the sensor performance can be ignored. Furthermore, we also studied the mechanical stability after bending the devices for hundreds of cycles and the corresponding results are depicted in Fig. S2. After bending for 5000 cycles, there is slight change in the conductance of the flexible devices, revealing the outstanding long-term and mechanical stability of these devices.

\section{CONCLUSION}

In summary, we designed and constructed a flexible multifunctional sensing system by integrating three NW photodetectors, one NW gas sensor with one microwire temperature sensor. The integrated system can monitor the environmental signals, such as detecting the concentration of $\mathrm{NH}_{3}$ gas through the gas sensing part, de-
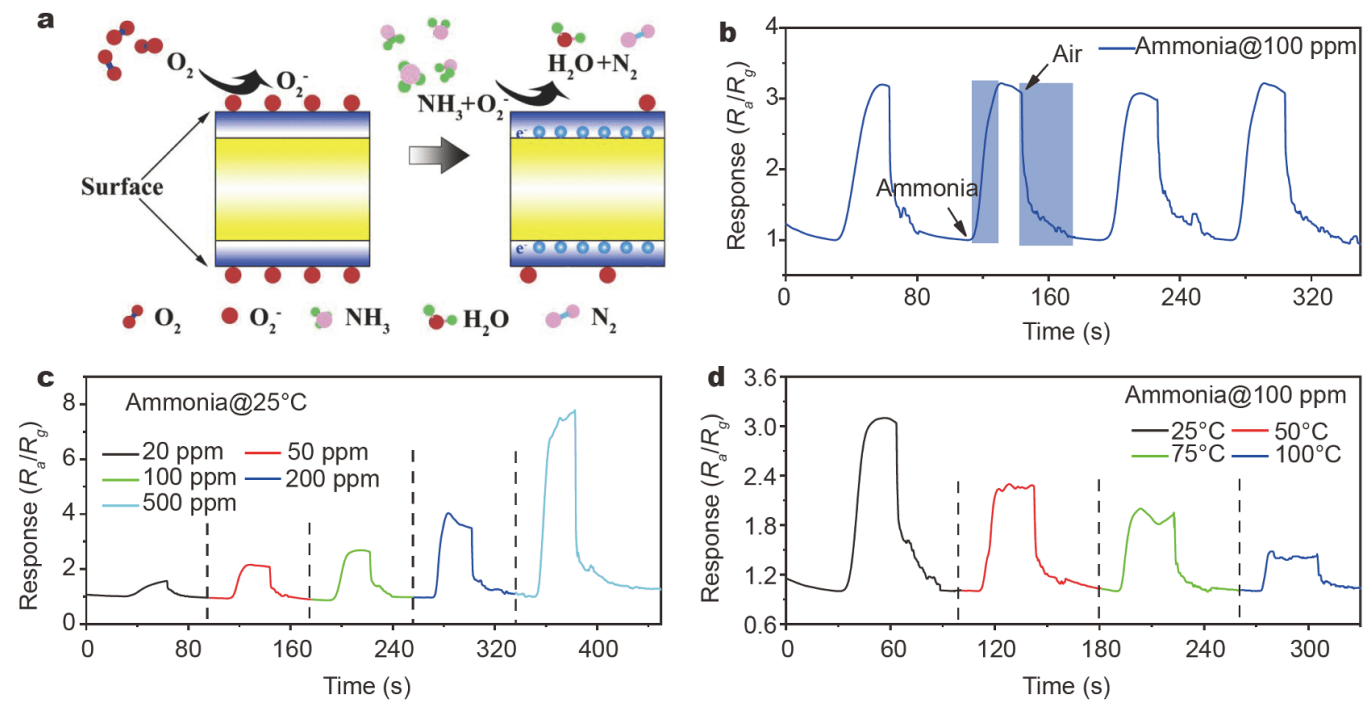

Figure 6 (a) Schematic of the sensing mechanism of $\mathrm{SrGe}_{4} \mathrm{O}_{9} \mathrm{NW}$-based gas sensor. (b) Cyclic response curves of the $\mathrm{SrGe}_{4} \mathrm{O}_{9} \mathrm{NW}$ sensor under $100 \mathrm{ppm}$ of $\mathrm{NH}_{3}$ at room temperature. (c) Response curves of the $\mathrm{SrGe}_{4} \mathrm{O}_{9} \mathrm{NW}$ sensor toward $\mathrm{NH}_{3}$ with different concentrations at room temperature. (d) Time-resolved response of the $\mathrm{SrGe}_{4} \mathrm{O}_{9} \mathrm{NW}$ device towards $100 \mathrm{ppm} \mathrm{NH}$ under different environment temperatures. 
tecting the brightness of light through the photodetectors and monitoring the environmental temperature through the temperature sensing part. More importantly, the linear relationship between the sensors and temperature was observed, making it possible to compensate the effect of temperature for practical applications. Our work demonstrates the great potential of the integrated system in the next-generation wearable electronics for healthcare applications.

\section{Received 5 March 2020; accepted 28 April 2020;} published online 9 July 2020

1 Bandodkar AJ, Jeerapan I, Wang J. Wearable chemical sensors: present challenges and future prospects. ACS Sens, 2016, 1: 464482

2 Kim YH, Kim SJ, Kim YJ, et al. Self-activated transparent allgraphene gas sensor with endurance to humidity and mechanical bending. ACS Nano, 2015, 9: 10453-10460

3 Lee H, Choi TK, Lee YB, et al. A graphene-based electrochemical device with thermoresponsive microneedles for diabetes monitoring and therapy. Nat Nanotech, 2016, 11: 566-572

4 Trung TQ, Ramasundaram S, Hwang BU, et al. An all-elastomeric transparent and stretchable temperature sensor for body-attachable wearable electronics. Adv Mater, 2016, 28: 502-509

5 Wang L, Chen S, Li W, et al. Grain-boundary-induced drastic sensing performance enhancement of polycrystalline-microwire printed gas sensors. Adv Mater, 2019, 31: 1804583

6 Wang K, Lou Z, Wang L, et al. Bioinspired interlocked structureinduced high deformability for two-dimensional titanium carbide (MXene)/natural microcapsule-based flexible pressure sensors. ACS Nano, 2019, 13: 9139-9147

7 Choi SJ, Yu H, Jang JS, et al. Nitrogen-doped single graphene fiber with platinum water dissociation catalyst for wearable humidity sensor. Small, 2018, 14: 1703934

8 Lou Z, Chen S, Wang L, et al. Ultrasensitive and ultraflexible eskins with dual functionalities for wearable electronics. Nano Energy, 2017, 38: 28-35

9 Lou Z, Wang L, Jiang K, et al. Programmable three-dimensional advanced materials based on nanostructures as building blocks for flexible sensors. Nano Today, 2019, 26: 176-198

10 Son D, Lee J, Qiao S, et al. Multifunctional wearable devices for diagnosis and therapy of movement disorders. Nat Nanotech, 2014, 9: 397-404

11 Yao S, Zhu Y. Wearable multifunctional sensors using printed stretchable conductors made of silver nanowires. Nanoscale, 2014, 6: 2345-2352

12 Choi S, Lee H, Ghaffari R, et al. Recent advances in flexible and stretchable bio-electronic devices integrated with nanomaterials. Adv Mater, 2016, 28: 4203-4218

13 Harada S, Honda W, Arie T, et al. Fully printed, highly sensitive multifunctional artificial electronic whisker arrays integrated with strain and temperature sensors. ACS Nano, 2014, 8: 3921-3927

14 Cai G, Wang J, Lee PS. Next-generation multifunctional electrochromic devices. Acc Chem Res, 2016, 49: 1469-1476

15 Gong S, Schwalb W, Wang Y, et al. A wearable and highly sensitive pressure sensor with ultrathin gold nanowires. Nat Commun, 2014, 5: 3132
16 Lou Z, Shen G. Flexible photodetectors based on 1D inorganic nanostructures. Adv Sci, 2016, 3: 1500287

17 Lou Z, Li L, Shen G. In $\mathrm{IaO}_{3}(\mathrm{ZnO})$ superlattice nanowires for high-performance ultraviolet photodetectors. Adv Electron Mater, 2015, 1: 1500054

18 Li RZ, Hu A, Zhang T, et al. Direct writing on paper of foldable capacitive touch pads with silver nanowire inks. ACS Appl Mater Interfaces, 2014, 6: 21721-21729

19 Yeom C, Chen K, Kiriya D, et al. Large-area compliant tactile sensors using printed carbon nanotube active-matrix backplanes. Adv Mater, 2015, 27: 1561-1566

20 Chai R, Lou Z, Shen G. Highly flexible self-powered photodetectors based on core-shell Sb/CdS nanowires. J Mater Chem C, 2019, 7: 4581-4586

21 Fang $\mathrm{H}, \mathrm{Hu} \mathrm{W}$, Wang P, et al. Visible light-assisted high-performance mid-infrared photodetectors based on single InAs nanowire. Nano Lett, 2016, 16: 6416-6424

22 Kwon DK, Porte Y, Ko KY, et al. High-performance flexible ZnO nanorod UV/gas dual sensors using Ag nanoparticle templates. ACS Appl Mater Interfaces, 2018, 10: 31505-31514

23 Lou Z, Yang X, Chen $\mathrm{H}$, et al. Flexible ultraviolet photodetectors based on $\mathrm{ZnO}-\mathrm{SnO}_{2}$ heterojunction nanowire arrays. J Semicond, 2018, 39: 024002

24 Lou Z, Li L, Shen G. High-performance rigid and flexible ultraviolet photodetectors with single-crystalline $\mathrm{ZnGa}_{2} \mathrm{O}_{4}$ nanowires. Nano Res, 2015, 8: 2162-2169

25 Zhang K, Luo T, Chen $\mathrm{H}$, et al. Au-nanoparticles-decorated $\mathrm{Sb}_{2} \mathrm{~S}_{3}$ nanowire-based flexible ultraviolet/visible photodetectors. J Mater Chem C, 2017, 5: 3330-3335

$26 \mathrm{Li} \mathrm{L}$, Lou Z, Chen $\mathrm{H}$, et al. Stretchable $\mathrm{SnO}_{2}$-CdS interlacednanowire film ultraviolet photodetectors. Sci China Mater, 2019, 62: 1139-1150

27 Wang $\mathrm{C}$, Xia K, Wang $\mathrm{H}$, et al. Advanced carbon for flexible and wearable electronics. Adv Mater, 2019, 31: 1801072

28 Gao Z, Jiang K, Lou Z, et al. Water-proof and thermally inert flexible pressure sensors based on zero temperature coefficient of resistance hybrid films. J Mater Chem C, 2019, 7: 9648-9654

29 Zhao L, Wang K, Wei W, et al. High-performance flexible sensing devices based on polyaniline/MXene nanocomposites. InfoMat, 2019, 1: 407-416

30 Deng J, Zhang R, Wang L, et al. Enhanced sensing performance of the $\mathrm{Co}_{3} \mathrm{O}_{4}$ hierarchical nanorods to $\mathrm{NH}_{3}$ gas. Sens Actuat B-Chem, 2015, 209: 449-455

Acknowledgements This work was supported by the National Natural Science Foundation of China (61874111 and 61625404), the Young Elite Scientists Sponsorship Program by CAST (2018QNRC001) and China Postdoctoral Science Foundation (2016M601131).

Author contributions The paper was written through contributions of all authors. All authors have given approval to the final version of the paper.

Conflict of interest The authors declare no conflict of interest.

Supplementary information Supporting data are available in the online version of the paper. 


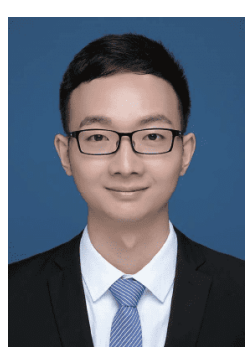

Haoran Chen received his BE degree in 2015 from the University of Science and Technology Beijing. He is a $\mathrm{PhD}$ student at the Institute of Semiconductors, Chinese Academy of Sciences. His research interests mainly focus on nanomaterials and optoelectronic devices.

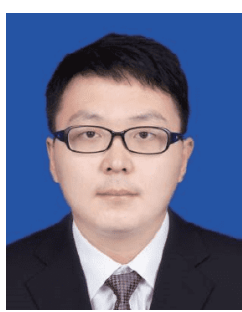

Zheng Lou is an associate professor at the Institute of Semiconductors, Chinese Academy of Sciences. He received his BSc degree (2009) and $\mathrm{PhD}$ degree (2014) from Jilin University. His current research focuses on the flexible electronics based on low-dimensional materials, including pressure sensors, electronic-skin, transistors and photo-detectors.

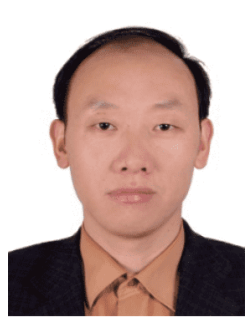

Guozhen Shen received his BSc degree (1999) in chemistry from Anhui Normal University and $\mathrm{PhD}$ degree (2003) in chemistry from the University of Science and technology of China. He joined the Institute of Semiconductors, Chinese Academy of Sciences as a professor in 2013. His current research focuses on the flexible electronics and printable electronics, including transistors, photodetectors, sensors and flexible energy storage and conversion devices.
一种用于监测多种环境信号的柔性多功能传感 系统

陈浩然 ${ }^{1 \dagger}$, 娄正 ${ }^{1,2 \dagger}$, 沈国震 ${ }^{*}$

摘要 随着便携式设备和物联网技术的飞速发展, 对系统可穿戴性 和集成性的要求加快了柔性多功能传感器的研究. 本文以半导体 纳米线为传感材料, 制备了四种平面器件, 包括光电探测器和气体 传感器. 由于纳米线具有大的表面体积比以及良好的亚波长效应, 所研制的基于纳米线的传感器都表现出优异的传感响应, 以及灵 敏度与温度间良好的线性关系. 将这些传感器与基于 $\mathrm{Ni}$ 微米线的 柔性温度传感器集成在一个柔性平台上, 构建了一种可穿戴的多 功能传感系统. 该系统可以同时检测多种环境参数, 包括温度变 化、从紫外可见光到近红外的光强变化、有害气体浓度等. 我们 所设计的柔性多功能传感系统为新兴的便携式、可穿戴电子产品 提供了一条新的思路. 